\title{
Experimental Analysis on Variation of the Amount of Particles Passing Through Traveling Wave Direct Energy Converter ${ }^{*)}$
}

\author{
Kazuhiro SHIBATA, Hiromasa TAKENO, Kazuya ICHIMURA, Satoshi NAKAMOTO \\ and Yousuke NAKASHIMA ${ }^{1)}$ \\ Graduate School of Engineering, Kobe University, Kobe 657-8501, Japan \\ 1) Plasma Research Center, University of Tsukuba, Tsukuba 305-8577, Japan
}

(Received 27 December 2018 / Accepted 22 March 2019)

\begin{abstract}
Traveling wave direct energy converter (TWDEC) was proposed as an efficient energy recovery device for 14.7 $\mathrm{MeV}$ protons produced by $\mathrm{D}_{-}{ }^{3} \mathrm{He}$ fusion reaction. Protons are velocity-modulated and bunched up, then induce traveling wave field which traps and decelerates themselves. In simulation experiments, it was found that the amount of particles was larger during application of modulation/deceleration field in the condition providing high efficiency than other conditions. This paper treats the phenomenon and will present results of analytical experiments and discussion. We found that ions were scattered due to modulation, and variation of the number of ions was corresponding to variation of deceleration effect. The possible mechanism of the phenomenon is scattering of particles due to bunching and its suppression due to trapping by deceleration field. In other words, high efficiency would be obtained by suppression of scattering.
\end{abstract}

(C) 2019 The Japan Society of Plasma Science and Nuclear Fusion Research

Keywords: TWDEC, constant deceleration scheme, dual-frequency modulation, scattering, particle trapping

DOI: $10.1585 /$ pfr. 14.3405078

\section{Introduction}

In $\mathrm{D}^{3} \mathrm{He}$ fusion power generation, most of energy produced by fusion reaction is carried as kinetic energy of charged particles such as electrons, thermal ions, and high energy protons of $14.7 \mathrm{MeV}$. The proton energy cannot be treated by conventional electrostatic converters due to technical limitations. To overcome this problem, application of a traveling wave direct energy converter (TWDEC) [1] is expected. Direct energy converters (DECs), which are made up of a cusp-type direct energy converter (CuspDEC) [2] and a TWDEC, were proposed as efficient energy recovery devices for $\mathrm{D}^{3}{ }^{3} \mathrm{He}$ fusion reaction in the previous research [3].

The charged particles from a reactor are incident into DECs, the energy of electrons and thermal ions are processed in CuspDEC and that of protons are in TWDEC. The CuspDEC is placed in the upstream, and designed to discriminate thermal ions and electrons from incident plasma. These particles are gathered into collectors, and those energy is converted into DC power. TWDEC is designed based on inverse process of a linear accelerator, and works for energy recovery from $14.7 \mathrm{MeV}$ protons. It consists of a modulator and a decelerator. Incident protons are introduced into the modulator and bunched up in the downstream by axial velocity modulation using RF electric field. The decelerator is located at the bunching point

author's e-mail: takeno@eedept.kobe-u.ac.jp

*) This article is based on the presentation at the 27th International Toki Conference (ITC27) \& the 13th Asia Pacific Plasma Theory Conference (APPTC2018). and bunched protons excite an electrostatic traveling wave. The wave is designed to be decelerated and traps protons by its potential well so that trapped protons are decelerated. Reduced kinetic energy of the protons are converted into the RF energy through an external transmission circuit connected to the decelerator.

In previous research on modulation process, numerical calculation predicted a possibility of ion scattering due to bunching [4]. Actually, a phenomenon suggesting ion scattering was found in simulation experiments [5]. In such a situation, some ions go out of the system and their energy is lost. Thus, cause and influence on deceleration of the phenomenon should be investigated [6]. In this work, we performed experimental analysis on radial scattering of ions by using an array of concentric electrodes, and investigated influence on deceleration process in various deceleration experiments.

In Sec. 2, the experimental setup and evaluation methods are shown. Overview of the TWDEC simulator and experimental procedure are explained. Measurement devices and evaluation are also explained. In Sec. 3, experimental results are shown, and discussion is also presented. In Sec. 4, conclusion of the paper is given.

\section{Experimental Step}

\subsection{TWDEC simulator}

A schematic view of the experimental simulator is shown in Fig. 1. The device consists of sections of ion source, extraction, modulation, deceleration, and measure- 


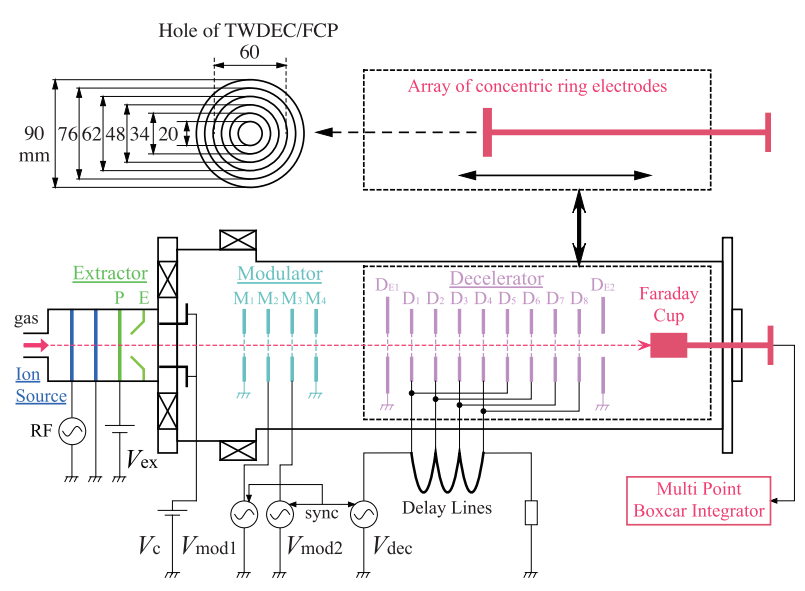

Fig. 1 Experimental simulator.

ment. These sections are placed coaxially.

In the ion source section, a helium plasma is generated by supplying an RF power of $15 \mathrm{MHz}$ to an RF antenna. The discharge is assisted by magnetic field created by two magnetic coils shown in Fig. 1. The discharge is performed by repetitive pulse operation, and the pulse has a frequency of $667 \mathrm{~Hz}$ and $67 \%$ duty ratio.

In the extraction section, extraction voltage $V_{\text {ex }}$ is applied to an extraction electrode at the end of the ion source. In this paper, $V_{\mathrm{ex}}=1.6 \mathrm{kV}$ and $3.2 \mathrm{kV}$ are used. The extracted ions are bundled into a beam shape by applying $V_{\mathrm{c}}$ to a cylindrical convergence electrode placed in the downstream of the extraction electrode.

In the modulator, we adopted the dual-frequency modulation method to improve deceleration effect. The dualfrequency modulation was proposed as favourable modulation method to improve ion bunching [7,8]. Actual structure of the modulator has four coaxial disk electrodes with a hole of $6 \mathrm{~cm}$ diameter covered by mesh. A sinusoidal wave voltage of $7 \mathrm{MHz}\left(V_{\bmod 1}\right)$ and synchronized one of $14 \mathrm{MHz}$ with leading phase difference of $0.5 \pi \mathrm{rad}$ $\left(V_{\bmod 2}\right)$ are applied to the second $\left(\mathrm{M}_{2}\right)$ and the third $\left(\mathrm{M}_{3}\right)$ electrodes, respectively, and other electrodes are grounded. The axial intervals between electrodes are the same as the running length of the ions in quarter period of $7 \mathrm{MHz}$. The effect of dual-frequency modulation was confirmed as generation characteristics of the third harmonic component [8] and variation of energy distribution function [9] in the previous papers.

For deceleration experiment in the simulator, formation of traveling wave in the decelerator is achieved by application of external RF voltages $\left(V_{\mathrm{dec}}\right)$ instead of induction due to bunched ions. This is called 'active decelerator' [10]. In the actual experiments, two function generators synchronized with each other are used. Those signals are pulse-modulated and amplified as $V_{\bmod 1}$ and $V_{\text {dec }}$. The phase of the signals can be controlled independently, and that for $V_{\text {mod1 }}$ is zero. As for $V_{\text {dec }}$, phases of $\theta_{\mathrm{dec}}=n \pi / 4(n=0,1, \ldots, 7)$ are settled for examination of deceleration effect for each experimental condition. We designed the decelerator based on the constant deceleration scheme [11]. In this scheme, traveling wave with constant deceleration traps ions in its potential well and trapped ions are decelerated together. The axial position of electrodes corresponding to $0.5 \pi$ phase interval can be determined by deceleration $\alpha$ according to $V_{\mathrm{ex}}$. For $V_{\mathrm{ex}}=1.6 \mathrm{kV}$ and $3.2 \mathrm{kV}$, we take $\alpha=5.5 \times 10^{11} \mathrm{~m} / \mathrm{s}^{2}$ and $7.8 \times 10^{11} \mathrm{~m} / \mathrm{s}^{2}$, respectively, and 6 and 8 electrodes are placed, respectively. The first electrode is at the bunching position. The structure of the electrodes are the same as that in the modulator. Each $0.5 \pi$ lagging voltage can be obtained by an RF source of $V_{\text {dec }}$ of $7 \mathrm{MHz}$ synchronized with $V_{\bmod 1}$ and delay lines.

\subsection{Measurement and evaluation}

At the end of the device, a Faraday cup (FCP) is installed which consists of an ion repeller grid (IR), a secondary electron repeller grid (SER) and an ion collector electrode (C). The entrance hole of the cup has also grid, and the size of the hole as well as those of the holes of IR and SER are the same diameter of $6 \mathrm{~cm}$ with those of TWDEC electrodes. The ions are discriminated for each energy by applying sweeping voltage $\left(V_{\mathrm{IR}}\right)$ to the IR. In the experiment, collector current $I_{\mathrm{c}}$ discriminated for each energy is obtained. The distribution function of the ions $f(E)$ is given by $f(E)=V_{\mathrm{IR}}^{-1 / 2}\left(\mathrm{~d} I_{\mathrm{c}}\right) /\left.\left(\mathrm{d} V_{\mathrm{IR}}\right)\right|_{V_{\mathrm{IR}}=E / e}$, where $E$ and $e$ mean ion energy and unit charge, respectively. The average energy of the ions are given by $\langle E\rangle=$ $\int_{0}^{\infty} E \cdot f(E) \mathrm{d} E / \int_{0}^{\infty} f(E) \mathrm{d} E$. We used deceleration rate defined by $R_{\mathrm{dec}}=\left(\left\langle E_{0}\right\rangle-\langle E\rangle\right) /\left\langle E_{0}\right\rangle$ for evaluation of deceleration effect, where $\left\langle E_{0}\right\rangle$ means the average energy of incident ions without modulation and deceleration.

An array of concentric ring electrodes used for measurement of radial distribution of ions is installed at the end of TWDEC simulator instead of the Faraday cup. Its structure is shown in Fig. 1. The array consists of six ring electrodes which are $\mathrm{P}_{1}-\mathrm{P}_{6}$ counting from the center. The size of each electrodes is shown in the figure. The electrodes are placed by removing the decelerator and the FCP, and the electrode array is axially movable to obtain axial variation of the radial distribution.

In the experiment, a multi-point boxcar integrator (MPB) was used to obtain time variation of energy distribution. The MPB was developed by authors [12]. The tim-

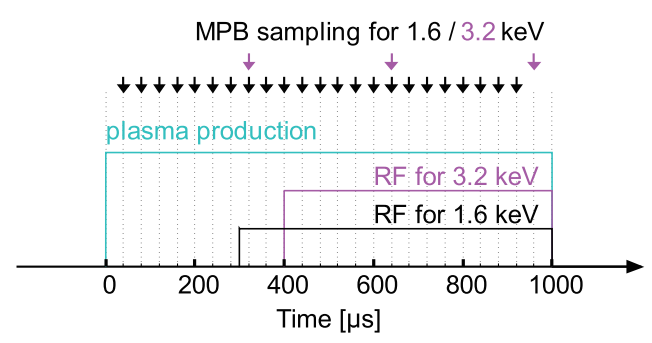

Fig. 2 Timing chart of RF $\left(V_{\bmod 1}, V_{\mathrm{mod} 2}, V_{\mathrm{dec}}\right)$ applications and MPB sampling. 
ing chart of RF applications and sampling by MPB in the actual experiment are shown in Fig. 2. The sampling timing is determined by taking period of steady state of plasma production and sweeping energy range into account. For $V_{\text {ex }}=1.6 \mathrm{kV}, V_{\bmod 1 / 2}$ and $V_{\text {dec }}$ are in $300-1000 \mu$ s from the start of plasma production, so every $40 \mu$ s during 320 $920 \mu \mathrm{s}$ are taken. For $V_{\mathrm{ex}}=3.2 \mathrm{kV}, 320,640$, and $960 \mu \mathrm{s}$ are taken for 400 - $1000 \mu \mathrm{s}$ RF application.

\section{Results \& Discussion}

\subsection{Radial behavior of ions}

Ion beam currents were measured by the concentric ring electrodes. In order to discuss the variation of radial distribution due to beam modulation, the results are summarized as in Fig. 3. Note that the abscissa $(L)$ means axial position of the array of concentric ring electrodes based on bunching position, and ordinate $\left(R_{\mathrm{c}}\right)$ means ratio of the modulated beam current relative to the corresponding current without modulation.

The current measured by $\mathrm{P}_{1}$ of the most inner electrode is consistently smaller than 1 . Those of $\mathrm{P}_{2}$ and $\mathrm{P}_{3}$ change a little around 1 for all axial positions. That of $\mathrm{P}_{4}$ decreases in the downstream of bunching point. For $\mathrm{P}_{5}$, all currents except at $L=30 \mathrm{~mm}$ are over 1 . That of $\mathrm{P}_{6}$ of the most outer electrode is consistently larger than 1.2, and larger values are found around bunching point. When we summarize roughly, internal components of the ion beam decrease due to modulation while external ones increase, and the variation is large in the downstream of the bunching point. Thus, it can be said that ions are scattered by field of ions bunched by modulation.

\subsection{Time variation of the amount of particles}

An example of the energy distribution of the ion beam measured by the FCP is shown in Fig. 4. The energy distribution of the beam is originally as that by black curve, and becomes as that by red curve due to deceleration. In spite of the condition of $V_{\mathrm{ex}}=1.6 \mathrm{kV}$, the original beam has a peak at $1.8 \mathrm{keV}$. This energy difference may be due to potential of the plasma in the ion source. The difference of initial beam energy affects deceleration process in the designed decelerator. However, the present difference $(0.2 \mathrm{keV})$ is almost the same as that by modulation voltage $(190 \mathrm{~V})$, so the particles can be trapped with bouncing motion in the potential well of traveling wave. From this result, we can calculate the number of ions by integrating the energy distribution function: $\int f(E) \mathrm{d} E$. Time evolution of the energy distribution is obtained by MPB sampling in one measurement, so time evolution of the number of ions is also obtained.

The time variation of the number of ions of $1.6 \mathrm{keV}$ ion beam is shown in Fig. 5. The number of modulated particles is denoted by black squares, and those of decelerated particles are denoted by red triangles for $\theta_{\mathrm{dec}}=5 \pi / 4$ when $R_{\mathrm{dec}}=39.1 \%$ and blue inverse triangles for $\theta_{\mathrm{dec}}=3 \pi / 4$

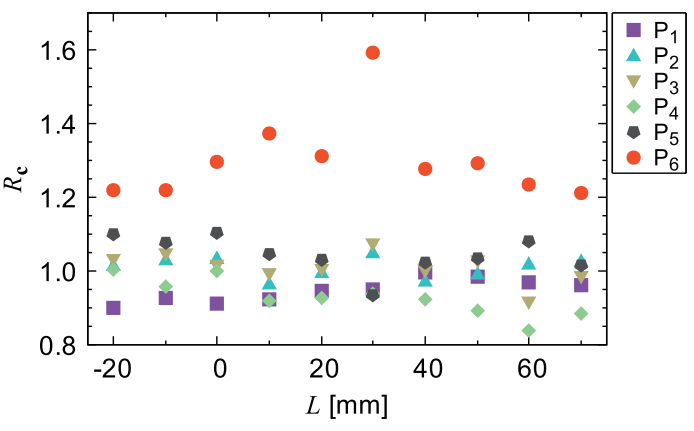

Fig. 3 Ratio of the modulated beam current relative to that without modulation for each ring electrode $\left(\mathrm{P}_{1}-\mathrm{P}_{6}\right)$.

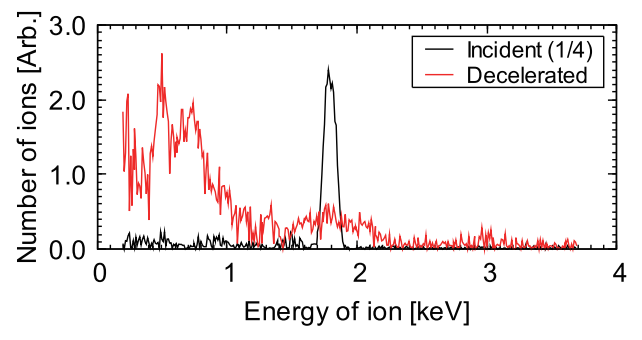

Fig. 4 Example of energy distribution.

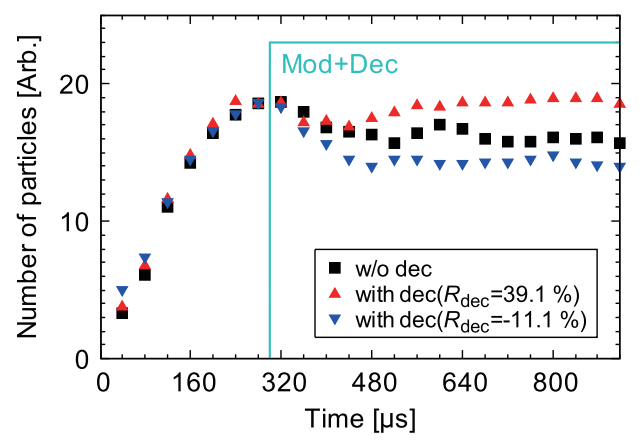

Fig. 5 Time variation of the number of ions of $1.6 \mathrm{keV}$ ion beam.

when $R_{\mathrm{dec}}=-11.1 \%$. The number of particles increases as time elapses from $40 \mu$ s to $280 \mu$ s due to plasma production. During RF application from $300 \mu$ s to $920 \mu$ s, the number of ions decreases without deceleration. In the case with deceleration of high $R_{\mathrm{dec}}$, decrement of the number of ions is suppressed and the same level before RF application is recovered around $700 \mu \mathrm{s}$. In the case with deceleration of low $R_{\mathrm{dec}}$, however, decrement of the number of ions is enhanced and the level is lower than without deceleration.

The time variation of the number of the ions of 3.2 $\mathrm{keV}$ ion beam is also examined, and is shown in Fig. 6 by using the same symbols as those in Fig. 5 (high/low $R_{\text {dec }}$ are obtained by different $\theta_{\mathrm{dec}}$ ). In the three conditions, the number of ions is the same with each other at $300 \mu$ s. After RF application, the number of ions without deceleration increases a little. In the condition of high $R_{\mathrm{dec}}$, the number of ions is larger than without deceleration, while it is less in the low $R_{\mathrm{dec}}$ condition. 


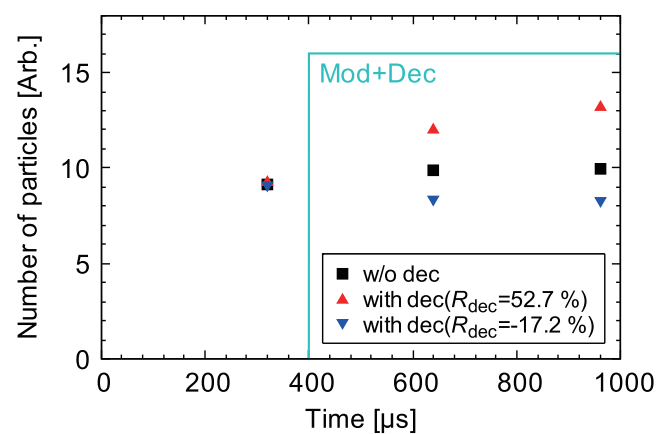

Fig. 6 Time variation of the number of ions of $3.2 \mathrm{keV}$ ion beam.

By applying modulation, spatially gathered ions repel each other, and are scattered. Thus, the number of ions in the beam path limited by the size of the holes of TWDEC electrodes decreases, and that introduced into the FCP with the same hole size decreases.

\subsection{Relation between the number of ions and deceleration effect}

We examined relation between variation of the number of ions and deceleration rate in the conditions of $V_{\mathrm{dec}}$ $=150,180,220 \mathrm{~V}_{0 \mathrm{p}}\left(\mathrm{V}_{0 \mathrm{p}}\right.$ means zero-to-peak voltage $)$. When we vary $\theta_{\mathrm{dec}}, R_{\mathrm{dec}}$ changes even in the same $V_{\mathrm{dec}}$ condition. The results of $V_{\mathrm{ex}}=1.6 \mathrm{kV}$ are summarized in Fig. 7. Note that the ordinate of Fig. 7 means the number of ions on $920 \mu$ s relative to that on $280 \mu$ s in the same measurement. According to Fig. 7, a positive correlation is found between the number of ions and the deceleration rate in each $V_{\text {dec }}$ condition. The number of ions is large when the deceleration rate is high, and vice-versa.

We also examined relation between the deceleration rate and the number of ions for $V_{\mathrm{ex}}=3.2 \mathrm{kV}$, and the results are shown in Fig. 8. Figures 8 (a) and (b) show increase rate of the number of ions and the deceleration rate versus initial phase of traveling wave $\theta_{\mathrm{dec}}$, respectively. Note that the ordinate in Fig. 8 (a) shows increase rate of number of ions from $320 \mu$ s to $960 \mu$ s. The chained line shows the level without RF application which means the pure incident beam. In the modulation indicated by the blue solid line, the increase rate is smaller than the incident beam, which is considered to be due to ion scattering. The red squares are those of deceleration, and they roughly vary sinusoidally to $\theta_{\mathrm{dec}}$. The deceleration rate in Fig. 8 (b) changes from $-17 \%$ to $53 \%$ in accordance with the increase rate. The result in Fig. 8 also shows variation of the increase rate is corresponding to variation of the deceleration rate.

From those results, we can conclude there is a strong correlation between the number of ions and the deceleration rate. As for the mechanism of this correlation, we can consider as follows.

In the constant deceleration scheme, the decelerator is designed so that an electrostatic wave travels and is decel-

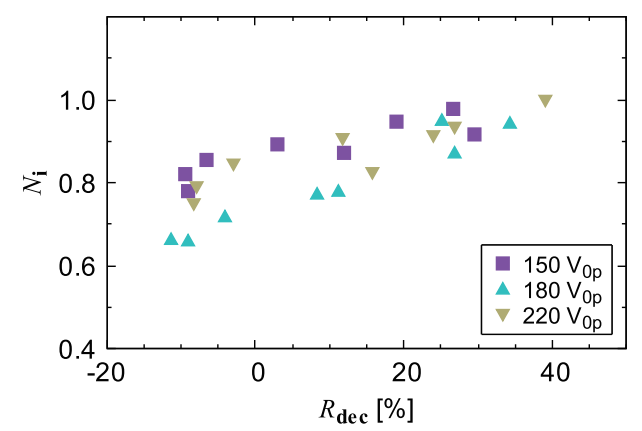

Fig. 7 Relative number of ions $\left(N_{\mathrm{i}}\right)$ versus deceleration rate $\left(R_{\mathrm{dec}}\right)\left(V_{\mathrm{ex}}=1.6 \mathrm{kV}\right)$.

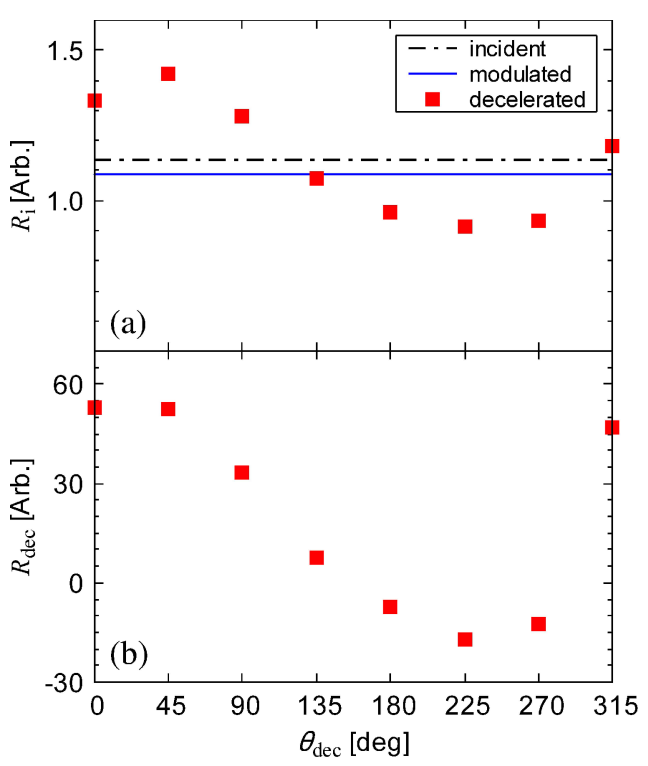

Fig. 8 Dependences on initial phase of traveling wave $\left(\theta_{\mathrm{dec}}\right)$ of (a) increase rate of the number of ions from $320 \mu$ s to $960 \mu \mathrm{s}\left(R_{\mathrm{i}}\right)$ and (b) deceleration rate $\left(R_{\mathrm{dec}}\right)$ for $3.2 \mathrm{keV}$ ion beam.

erated axially. Ions are trapped by the potential well of the traveling wave so that the trapped ions are decelerated. For deceleration, required trapping is in the axial direction, but the effect also restricts radial ion motion. The modulated ion beam is scattered by the field created by bunched ions, however, restriction by the trapping corrects ion orbits in the axial direction. So the number of ions in the beam column could be changed by application of deceleration field.

As TWDEC works based on the inverse process of a linear accelerator, the deceleration effect depends on the initial phase of the traveling wave $\theta_{\mathrm{dec}}$. In the phase of better effect, the number of trapped ions are more, which results in higher deceleration rate. On one hand, the trapping also affects radially, so the number of ions in the beam column increases. That's why the number of ions and the deceleration rate correlates.

As for scattering phenomenon, the existence of the phenomenon was just confirmed experimentally [5], and 
its detailed examination has not been done. Further simulation experiments, such as measurement at a larger radius are required. At the same time, the scattering phenomenon itself is possible to become an obstacle in the experiments without a decelerator. As an operation only with a modulator is nothing but a simulation experiment, influence of the scattering phenomenon can be relatively reduced by employing a thick ion beam.

\section{Conclusion}

We carried out experimental analysis on ion scattering in a TWDEC simulator. As a result, we found that ions moved outward due to bunching by modulation. Subsequently, deceleration experiments were performed, and it was found that number of ions changes having a correlation with deceleration effect. The number of ions increases in the preferred deceleration conditions while it decreases in the other conditions. Thus, it can be said that the ion scattering is restricted by the trapping effect by the potential well of traveling wave.

\section{Acknowledgment}

This work was supported in part by the bilateral co- ordinated research between PRC, Univ. Tsukuba, NIFS, and Kobe Univ. (NIFS13KUGM082), and was also supported in part by a Grant-in-Aid for Scientific Research (B) (16H04317) from JSPS.

[1] H. Momota, LA-11808-C, Los Alamos Natl. Lab., 8 (1990).

[2] H. Momota et al., Proc. 7th Int. Conf. on Emerging Nucl. Energy Systems 16 (1993).

[3] Y. Tomita et al., Nucl. Instrum. Methods Phys. Res. A, 402, 421 (1998).

[4] K. Nishimura et al., Trans. Fusion Sci. Technol. 63, 310 (2013).

[5] H. Takeno et al., Plasma Conference 2017, 24P-91 (2017).

[6] K. Shibata et al., Plasma Conference 2017, 24P-90 (2017).

[7] H. Sato et al., Joint meeting of 26th International Toki Conference and 11th Asia Plasma and Fusion Association Conference, P1-99 (2017).

[8] H. Takeno et al., Plasma Fusion Res. 14, 2405014 (2019).

[9] K. Shibata et al., Plasma Fusion Res. 14, 2405027 (2019).

[10] H. Takeno et al., Trans. JSASS Aerospace Tech. Jpn. 14, $\mathrm{Pb} \_105$ (2016).

[11] Y. Togo et al., Plasma Fusion Res. 10, 3405013 (2015).

[12] H. Takeno et al., 10th Joint Convention of Fusion Energy, 20-130 (2014) (in Japanese). 